\title{
The Chemical Roots of Iron-Sulfur Dependent Metabolism
}

\author{
Claudia Bonfio and Sheref S. Mansy*(1) \\ CIBIO, University of Trento, via Sommarive 9, 38123 Povo, Italy
}

Tron-sulfur clusters are among the most ancient biological 1 cofactors and are involved in a wide variety of functions in living cells. Despite the central role that iron-sulfur clusters play in metabolism, it has remained unclear if the prebiotic chemistry that led to life was shaped by the activity of ironsulfur catalysts. In the absence of a protein scaffold, iron(II) in sulfur-rich environments typically reacts to form insoluble polymeric structures. Such observations, in part, led to the hypothesis that the chemistry provided by iron-sulfur proteins in contemporary metabolism is derived from iron-sulfur precipitates found at the bottom of the ocean. For example, the arrangement of iron and sulfide in the mineral greigite is similar to that of $[3 \mathrm{Fe}-4 \mathrm{~S}]$ and $[4 \mathrm{Fe}-4 \mathrm{~S}]$ proteins. However, no hypothesis has been proposed for how enzyme-regulated metabolism could have emerged from such mineral-based chemistry. Recent work by Bonfio et al. ${ }^{1}$ suggests a plausible route toward protein catalysts by exploiting biologically relevant ligands for the formation of model prebiotic iron-sulfur clusters.

Three challenges that need to be overcome to synthesize prebiotic iron-sulfur clusters in aqueous solution include the identification of prebiotically plausible ligands, the oxidation state of the iron ions, and the availability of sulfide ions. Although decades of work by Holm and others have expertly probed the chemistry of iron-sulfur clusters, nearly all of these studies were performed in organic solvents. Recently, while investigating the cellular synthesis and transport of iron-sulfur clusters, the Cowan group revealed the ability of the tripeptide L-glutathione to coordinate a $[2 \mathrm{Fe}-2 \mathrm{~S}]$ cluster in aqueous solution. ${ }^{2}$ In this case, four L-glutathione molecules are required to coordinate a single $[2 \mathrm{Fe}-2 \mathrm{~S}]$ cluster with each peptide donating a single cysteinyl thiolate. Because prebiotic mechanisms for the polymerization of short peptides on the primitive Earth have been demonstrated, prebiotically available peptides may have been capable of stabilizing the formation of iron-sulfur clusters. Such a conclusion would be bolstered by demonstrating a lack of sequence specificity for iron-sulfur coordination. If only a small fraction of tripeptide sequences showed the ability to coordinate an iron-sulfur cluster, then the likelihood of iron-sulfur clusters coordinated by peptides on the prebiotic Earth would be low. Instead, Bonfio et al. demonstrated that $>90 \%$ of the 35 tripeptides tested were competent for iron-sulfur coordination, suggesting that cysteine-containing tripeptide sequence space is predisposed to the coordination of an iron-sulfur cluster. This is important because contemporary metalloproteins often rely on the intrinsic properties of the metallocofactor for activity, from the binding of nucleic acids ${ }^{3}$ to the catalysis of metabolic reactions. In other words, the needed chemistry is more reliant on the metallocofactor than on the protein scaffold.

Because of the anoxic conditions of the prebiotic Earth, iron ions are believed to have been predominantly present as
iron(II). However, [2Fe-2S] and [4Fe-4S] clusters invariably contain at least one iron(III) center. The observation of fully reduced $[2 \mathrm{Fe}-2 \mathrm{~S}]$ and $[4 \mathrm{Fe}-4 \mathrm{~S}]$ clusters, i.e., an all-ferrous state, is rare and requires a particularly rigid protein scaffold and reductants outside of the range found in biology. For example, [2Fe-2S] L-glutathione cannot be synthesized from iron(II) under strictly anaerobic conditions. It thus seems unlikely that iron-sulfur clusters could have been synthesized on the prebiotic Earth if only iron(II) had been present. However, the oxidation of iron ions is not solely dependent on the presence of oxygen. Instead, iron(II) can be efficiently photooxidized with ultraviolet (UV) light regardless of the presence of oxygen. In fact, a [2Fe-2S] cluster is assembled on L-glutathione upon the anaerobic addition of iron(II) in the presence of a UV light source that mimics the radiation that would have reached the prebiotic, ozone-free Earth from the young Sun (Figure 1). ${ }^{1}$

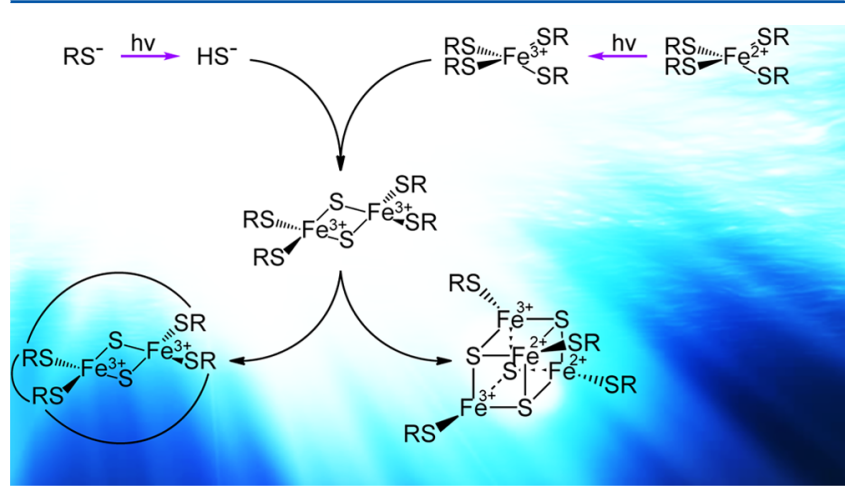

Figure 1. Iron-sulfur cluster synthesis is mediated by UV light. Photolysis and photooxidation in light-exposed aqueous regions lead to the synthesis of $[2 \mathrm{Fe}-2 \mathrm{~S}]$ and $[4 \mathrm{Fe}-4 \mathrm{~S}]$ clusters coordinated to cysteine-containing peptides. Initially, the iron-sulfur clusters are coordinated to short peptides ( $R$ ) that may subsequently undergo polymerization into longer ferredoxin-like polypeptides.

Since short peptides can stabilize the formation of ironsulfur clusters, and the needed iron(III) can be provided by sunlight, the only remaining piece of the puzzle is the availability of sulfide ions. Although sulfur is one of the most abundant elements in the universe, hydrogen sulfide has a low solubility and escapes from solution as a gas. Slightly alkaline conditions do lead to retention in an aqueous solution in the form of hydrosulfide $\left(\mathrm{HS}^{-}\right)$; however, insoluble iron hydroxide forms above $\mathrm{pH}$ 7. Nevertheless, the volatility of hydrogen sulfide can be avoided by trapping sulfur within organic thiols, if the organic thiol can deliver sulfide ions during cluster

Received: August 28, 2017 
synthesis. Here again, sunlight is the key ingredient. Exposure to UV light leads to the photolysis of cysteine and other thiolcontaining compounds, thereby releasing sulfide ions into solution. When the reaction is performed in the presence of iron ions and a cysteine-containing tripeptide, $[2 \mathrm{Fe}-2 \mathrm{~S}]$ and [4Fe-4S] clusters emerge.

The described chemistry can occur with organic thiols other than peptides. However, peptides are more attractive ligands, because scenarios can be envisaged with peptides that lead to the formation of more protein-like polypeptides. Computational studies suggest that the amino and carboxy termini of adjacent L-glutathione ligands coordinated to the same $[2 \mathrm{Fe}-$ $2 S$ ] cluster are physically proximal to each other. ${ }^{4}$ Therefore, a simple dehydration reaction, perhaps mediated by an RNA or peptide catalyst, could conceivably lead to polymerization into longer polypeptides. The templated polymerization of cysteinecontaining tripeptides would result in the formation of $\mathrm{CX}_{2} \mathrm{C}$ sequences, which is a common metal-binding motif that is observed in modern-day ferredoxins. Because [2Fe-2S] tripeptides are redox-active, catalytically active, ferredoxin-like sequences could potentially emerge from metal ion-templated polymerization of cysteine-containing tripeptide sequences. Similar scenarios are difficult to imagine for nonpeptide ligands.

Sunlight represents one of the Earth's largest energy sources and has been implicated in the prebiotic synthesis of amino acids, sugars, nucleotides, lipid precursors, ${ }^{5}$ and now ironsulfur clusters. ${ }^{1}$ Coupled with work on model protocells and the nonenzymatic copying of nucleic acids, experimental evidence is beginning to converge on a small subset of scenarios for the emergence of the Earth's first cells. That is, surface water conditions are the most compatible with the available experimental evidence. Of course, alternate theories exist, and no hypothesis is yet strong enough to explain all of the major transitional events from prebiotic chemistry to protocellular life. It will take more work from a variety of disciplines to further constrain the likely scenarios and to root laboratory chemistry experiments in geological settings. What is clear is that life as we know it depends on iron-sulfur clusters and that ancestral-like iron-sulfur peptides are easily formed in the presence of UV light.

\section{AUTHOR INFORMATION}

\section{Corresponding Author}

*E-mail: mansy@science.unitn.it.

\section{ORCID ${ }^{\circ}$}

Sheref S. Mansy: 0000-0003-2382-198X

\section{Funding}

We thank the Simons Foundation (290358) for funding.

\section{Notes}

The authors declare no competing financial interest.

\section{REFERENCES}

(1) Bonfio, C., Valer, L., Scintilla, S., Shah, S., Evans, D. J., Jin, L., Szostak, J. W., Sasselov, D. D., Sutherland, J. D., and Mansy, S. S. (2017) UV-light-driven prebiotic synthesis of iron-sulfur clusters. Nat. Chem., DOI: 10.1038/nchem.2817.

(2) Qi, W., Li, J., Chain, C. Y., Pasquevich, G. A., Pasquevich, A. F., and Cowan, J. A. (2012) Glutathione Complexed Fe-S Centers. J. Am. Chem. Soc. 134, 10745-10748.

(3) Tse, E. C. M., Zwang, T. J., and Barton, J. K. (2017) The Oxidation State of [4Fe4S] Clusters Modulates the DNA-Binding Affinity of DNA Repair Proteins. J. Am. Chem. Soc. 139, 12784.
(4) Scintilla, S., Bonfio, C., Belmonte, L., Forlin, M., Rossetto, D., Li, J., Cowan, J. A., Galliani, A., Arnesano, F., Assfalg, M., and Mansy, S. S. (2016) Duplications of an iron-sulphur tripeptide leads to the formation of a protoferredoxin. Chem. Commun. 52, 13456-13459.

(5) Patel, B. H., Percivalle, C., Ritson, D. J., Duffy, C. D., and Sutherland, J. D. (2015) Common origins of RNA, protein and lipid precursors in a cyanosulfidic protometabolism. Nat. Chem. 7, 301307. 